\title{
Exciton Level Structure and Dynamics in Tubular Porphyrin Aggregates
}

Yan Wan, ${ }^{1 \#}$ Anna Stradomska, ${ }^{2 \# \$}$ Sarah Fong, ${ }^{1}$ Zhi Guo, ${ }^{1,3}$ Richard D. Schaller, ${ }^{4,5}$ Gary P. Wiederrecht, ${ }^{4}$ Jasper Knoester, ${ }^{2 *}$ and Libai Huang ${ }^{1 *}$

${ }^{1}$ Radiation Laboratory, University of Notre Dame, Notre Dame, IN 46556, USA

${ }^{2}$ Zernike Institute for Advanced Materials, University of Groningen, Nijenborgh 4, 9747AG Groningen, The Netherlands

${ }^{3}$ Department of Aerospace and Mechanical Engineering, University of Notre Dame, Notre Dame, IN 46556, USA

${ }^{4}$ Center for Nanoscale Materials, Argonne National Laboratory, Argonne, IL 60439, USA

${ }^{5}$ Department of Chemistry, Northwestern University, Evanston, IL 60208

\# Contributed equally

${ }^{\$}$ Current address: School of Chemistry, University of Glasgow

*Corresponding authors; e-mails: J.Knoester@rug.nl, lhuang2@nd.edu

${ }^{\&}$ Current address: Department of Chemistry, Purdue University 


\begin{abstract}
We present an account of the optical properties of the Frenkel excitons in self-assembled porphyrin tubular aggregates that represent an analog to natural photosynthetic antennae. Using a combination of ultrafast optical spectroscopy and stochastic exciton modeling, we address both linear and nonlinear exciton absorption, relaxation pathways, and the role of disorder. The static disorder-dominated absorption and fluorescence linewidths show little temperature dependence for the lowest excitons (Q band), which we successfully simulate using a model of exciton scattering on acoustic phonons in the host matrix. Temperature dependent transient absorption of and fluorescence from the excitons in the tubular aggregates are marked by non-exponential decays with time scales ranging from a few picoseconds to a few nanoseconds, reflecting complex relaxation mechanisms. Combined experimental and theoretical investigations indicate that nonradiative pathways induced by traps and defects dominate the relaxation of excitons in the tubular aggregates. We model the pump-probe spectra and ascribe the excited state absorption to transitions from one-exciton states to a manifold of mixed oneand two-exciton states. Our results demonstrate that while the delocalized Frenkel excitons (over 208 (1036) molecules for the optically dominant excitons in the Q (B) band) resulting from strong intermolecular coupling in these aggregates could potentially facilitate efficient energy transfer, fast relaxation due to defects and disorder probably present a major limitation for exciton transport over large distances.
\end{abstract}


Keywords: Frenkel exciton, biomimetic photosynthetic antennae, ultrafast spectroscopy, stochastic exciton modeling 


\section{Introduction}

The photosynthetic antenna is a universal component of the photosynthetic apparatus in bacteria, algae, and higher plants that absorbs light and transfers the excitation energy by nonradiative processes to the reaction center. ${ }^{1-4}$ A remarkable feature of all of these antenna systems is that light energy absorbed by any of the chromophores, within even the largest arrays that are hundreds of nanometers from the reaction center, is transferred to the reaction center with an overall internal quantum efficiency in excess of $90 \%{ }^{2}$ Supramolecular assemblies order large numbers of chromophores into structures that bridge length scales from nanometers to mesoscale dimensions, mimicking natural photosynthetic antennae. ${ }^{5-10}$ In the presence of strong intermolecular coupling, as is the case for J-aggregates, the chromophores can act collectively (coherently) and potentially support faster energy transport. ${ }^{4,7,11-15}$ The Frenkel exciton, a collective excitation shared by many molecules, serves as the best description of the excited states of these molecular aggregates. ${ }^{11,16-17}$

Tubular aggregates formed by self-assembly of dye molecules represent one of the most promising constructs for biomimetic photosynthetic antenna systems. ${ }^{5,7,18-20} \mathrm{~A}$ prominent example of tubular molecular aggregates is derived from meso-tetra(4-sulfonatophenyl) porphyrin (TPPS4) and provides a biomimetic analogue of the chlorosomes of green sulfur bacteria. ${ }^{9,21-24}$ The structure of TPPS4 tubular aggregates has been characterized by small angle X-ray scattering, atomic force microscopy (AFM), and cryo-electron microscopy. ${ }^{25-33}$ TPPS4 tubular aggregates are found to be cylindrical 
structures with a diameter of approximately $16-18 \mathrm{~nm}^{31,33}$ and a length up to several microns. As shown in Figure 1, these tubular aggregates can be viewed as a rolled-up 2D sheet of porphyrin molecules. ${ }^{31}$ The optical properties of these tubular aggregates have been investigated by both experimental and theoretical methods. ${ }^{31,34}$

To realize the potential of tubular aggregates as biomimetic photosynthetic antennae for efficient energy transport, it is crucial to probe and understand the level structure and dynamics of the excitons in these systems. Questions have remained about the degree of exciton localization, energy relaxation pathways and timescales, energy transport mechanisms, and the occurrence of superradiant and dark states. Although exciton dynamics have been investigated using various ultrafast spectroscopic methods, ${ }^{35-41}$ a systematic temperature-dependent study to elucidate the nature of radiative and nonradiative pathways has not yet been reported. In this paper, we report a detailed investigation of the energy level structure an the temperature dependent ultrafast relaxation processes of the Frenkel excitons in self-assembled TPPS4 tubular aggregates by employing temperature dependent ultrafast spectroscopy in combination with stochastic exciton modeling.

This paper is organized as follows. Section II describes the sample preparation and methods for carrying out the experiments and performing the theoretical modeling. The results of the temperature dependent steady-state and time-resolved absorption and fluorescence experiments are presented in section III. A discussion of the temperature-dependent absorption linewidth and the radiative and nonradiative relaxation 
of the excitons is given, along with numerical simulations of the experimental data using a Frenkel exciton model. Finally, the conclusions are presented in section IV.

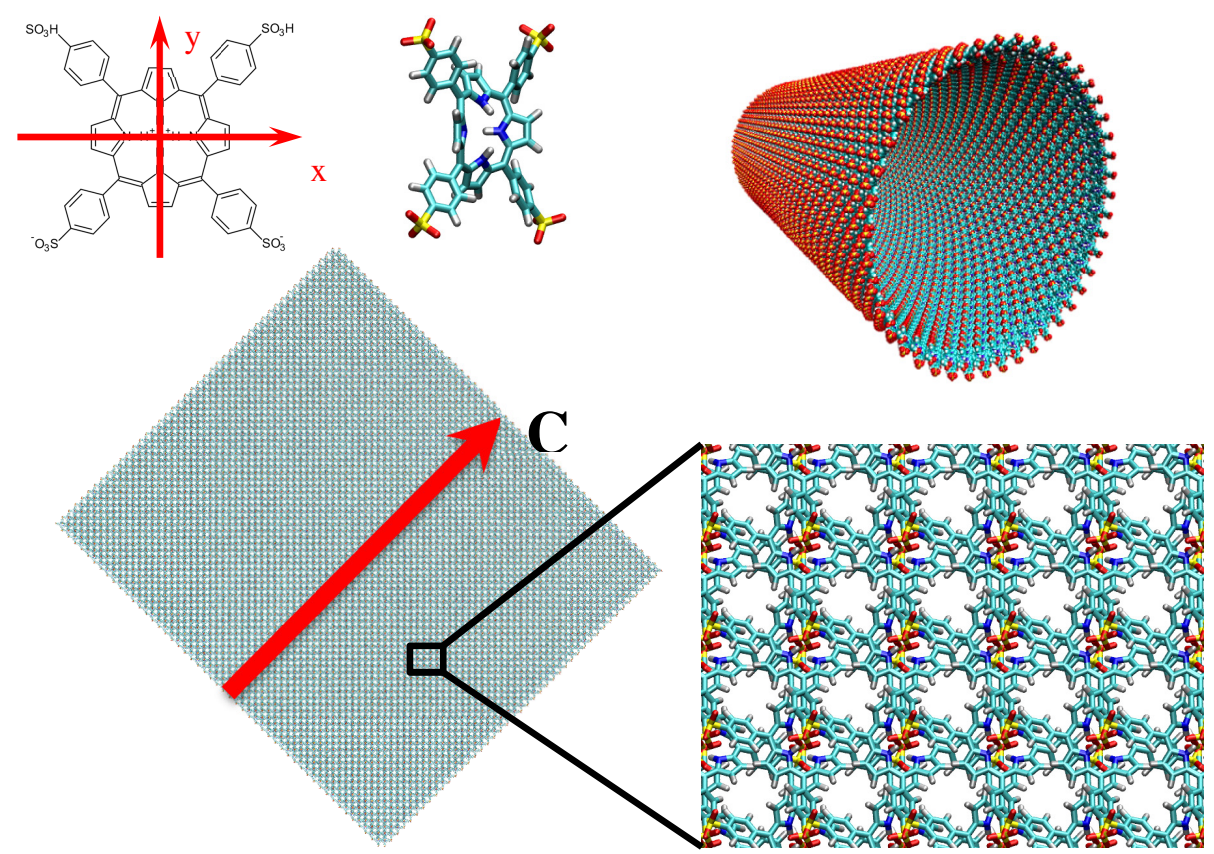

Figure 1. Chemical structure of the meso-tetra(4-sulfonatophenyl) porphyrin (TPPS4) monomer (top). The tubular aggregate forms at $\mathrm{pH} \sim 1 . \quad$ In order to obtain a cylindrical structure, a planar aggregate is seamlessly rolled onto the surface of the cylinder (bottom). The planar aggregate is rolled along the chiral vector $\mathrm{C}$ of $(43,43)$ (in units of the lattice constants), in such a way that the chiral vector becomes the circumference of the cylinder. ${ }^{31}$

\section{Methods}

\section{II.1 Experimental details}

Sample preparation: we followed the established procedure to prepare TPPS4 tubular aggregates. $^{25}$ A stock solution of $1 \mathrm{mM}$ meso-tetra-4-sulfonatophenylporphine tetrasodium salt (Frontier Scientific, used without further purification) was obtained by dissolving in DI water. Ethanol (200 proof pure, KOPTEC) was used to dilute the stock solution to a final TPPS4 concentration of $20 \mu \mathrm{M}$. 
The tubular aggregates were subsequently formed by adding $\mathrm{HCl}$ solution (Fisher Scientific) at room temperature and letting the solution stand for at least 45 minutes allowing self-assembly. We verified that these are tubular structures by performing atomic force microscopy measurements after depositing them on a glass substrate, as shown in Figure S1 of the supporting information (SI). The optimized $\mathrm{HCl}$ concentration for aggregate formation was $0.035 \mathrm{M}$, corresponding to a $\mathrm{pH}$ value of $\sim 1$. The final ratio of ethanol: water was 97:3, which functions as a cryoprotectant for the low temperature measurements described below. For comparison, the monomer solution at the same concentration of $20 \mu \mathrm{M}$ was prepared in an ethylene glycol: water $(7: 3)$ mixture at $\mathrm{pH} \sim 4$. In the monomer case, the 7:3 ethylene glycol: water mixture serves as the cryoprotectant. Steady-state fluorescence and absorption spectroscopy: room temperature UV-vis absorption and fluorescence spectra were measured with a UV-vis spectrophotometer (Cary 50 Bio, Varian) and a fluorescence spectrophotometer (Fluorolog, Jobin Yvon Inc.), respectively. The sample for low temperature steady-state absorption and fluorescence spectroscopy measurements was kept in a liquid helium optical cryostat (Microstat He, Oxford Instruments). The sample was irradiated by the output of a Ti:Sapphire laser (Mira 900, Coherent) generating $150 \mathrm{fs}$ pulses at a repetition rate of $80 \mathrm{MHz}$ that synchronously pumped an optical parametric oscillator (Mira-OPO, Coherent). The fluorescence was recorded with a CCD camera (Newton, Andor Technology) coupled to an imaging spectrograph (Shamrock, Andor Technology).

Temperature-dependent fluorescence lifetime measurements: the laser system employed 
was a $1 \mathrm{kHz}$ repetition rate Ti:sapphire amplifier (Spectra Physics spitfire) which produced $\sim 50 \mathrm{fs}$ pulses. The pulses from the amplifier were parametrically converted with an optical parametric amplifier (TOPAS, Light Conversion). A streak camera coupled to a spectrometer was used to record spectrally resolved fluorescence lifetimes with a time resolution of $\sim 7 \mathrm{ps}$.

Temperature-dependent transient absorption spectroscopy: transient absorption experiments were performed with a Spectra Physics amplified Ti:Sapphire laser system coupled to a TOPAS optical parametric amplifier (Light Conversion). The samples were housed in a liquid helium optical cryostat. The TOPAS provided 130 fs pump pulses that were tunable from $450 \mathrm{~nm}-2000 \mathrm{~nm}$ at $1.6 \mathrm{KHz}$. The probe pulse was a coherent white light continuum generated by focusing a small fraction $(<1 \%)$ of the Ti: Sapphire amplifier output at $800 \mathrm{~nm}$ onto a $3 \mathrm{~mm}$ thick sapphire window. A femtosecond transient absorption spectrometer (Ultrafast System Helios) was employed for the data acquisition. The polarizations of the pump and probe beams were controlled independently by two half-wave plates. The time resolution for the transient absorption measurements was $\sim 150 \mathrm{fs}$. The low-temperature transient absorption and fluorescence lifetime measurements were carried out at the Center for Nanoscale Materials at the Argonne National Laboratory. The transient absorption and fluorescence spectra were analyzed with a global analysis program Glotaran developed at the Vrije Universiteit Amsterdam. ${ }^{42}$

\section{II.2 Theoretical Modeling}

We described the aggregate structure using a model geometry which was proposed in 
previous work $^{31}$ and was shown to reproduce the structure of the experimental absorption and linear dichroism spectra ${ }^{34}$. We refer the reader to the original papers for details, and only give a brief summary here. In order to obtain a cylindrical structure, a planar aggregate was seamlessly rolled onto the surface of the cylinder as shown in Figure 1. This planar aggregate was built on a square lattice, with lattice constant of $0.93 \mathrm{~nm}$, and contained one TPPS4 molecule per unit cell. The molecules were tilted out of plane, so that the positively charged core of each molecule was sandwiched between the negatively charged sulfonate groups of neighboring molecules. We obtained this tilting of molecules by two rotations: initially the molecules were oriented so that their long axes were parallel to the lattice directions, first they were rotated by $24^{\circ}$ around their first long axis $(\hat{x}+\hat{y})$, and subsequently they were rotated by $-24^{\circ}$ around their second long axis $(\hat{x}-$ $\hat{y})$. Finally, this planar aggregate was rolled along the chiral vector of $(43,43)$ (in units of the lattice constants) in such a way that the chiral vector became the circumference of the cylinder (Figure 1).

One-exciton states: we modeled the excited states of TPPS4 aggregates using a Holstein-type Hamiltonian, which takes into account four electronic excited states per monomer (the $Q_{x}, Q_{y}, B_{x}$, and $B_{y}$ states) linearly coupled to one effective harmonic vibration of frequency $\omega_{0}$, as described in section II of the SI. We explicitly took into account static disorder by adding uncorrelated random shifts, taken from a Gaussian distribution, to the molecular excitation energies. We numerically diagonalized this Hamiltonian within the one-particle approximation ${ }^{43}$ to obtain the collective excited 
states. Two of us have shown previously ${ }^{34}$, using a simplified model that disregarded the effects of the static disorder, that this approximation reproduces well the optically active one-exciton states of TPPS4 tubes.

Dynamics: to account for dynamical effects we included the interaction with a thermal bath of environmental degrees of freedom. We treated this dynamic environment using the Pauli Master Equation (PME) approach ${ }^{44-45}$, which describes the effects of intraband scattering between the aggregate's excited states induced by phonons in the environment (see also section II of the SI for details). We used the Debye spectral density (with an exponential cut-off), which corresponds to scattering on acoustic phonons in the environment and previously has been used successfully to model optical and energy transport properties of other molecular dye aggregates. ${ }^{46-47}$ The sum of the scattering rates $W_{l k}$ from state $k$ to all possible final states $l$ yields the dephasing rate of the $k^{\text {th }}$ state: $\gamma_{k}^{d e p h}=\frac{1}{2} \sum_{l \neq k} W_{l k}$

Linear absorption: the positions and oscillator strengths of the collective excited states followed from the numerical diagonalization of the Hamiltonian. The linewidths were obtained by accounting for radiative and nonradiative relaxation to the ground state, dephasing deriving from the intraband scattering, as well as the static disorder. To deal with the former three processes, each excited state $k$ contributes to the absorption spectrum a Lorentzian peak with half width at half maximum of $\Gamma_{k}=\frac{1}{2} \gamma_{k}^{r a d}+$ $\frac{1}{2} \gamma_{k}^{\text {non-rad }}+\gamma_{k}^{\text {deph }}$. The radiative rate $\gamma_{k}^{r a d}$ of each excited state is estimated based on its oscillator strength (see section II of SI for details). We assume that the nonradiative 
decay in the aggregate is dictated by the nonradiative rate of the individual molecules and not changed by aggregation; hence the nonradiative rate $\gamma_{k}^{n o n-r a d}$ equals the monomer nonradiative rate of $0.12 \mathrm{~ns}^{-1}$. Finally, the disorder is taken into account by averaging over 200 disorder realizations.

Pump-probe spectrum: we modeled this using the hard-core boson approach, which does not require calculating the two-exciton states; the latter would make it impossible to model systems as large as these porphyrin nanotubes ${ }^{48-49}$ Previously, the method has been applied to tubular aggregates with one transition per molecule. ${ }^{50}$ We generalized the method of ref. ${ }^{49}$ in order to treat the case with several electronic excited states per molecule. To make the calculations feasible, we neglected (only in these calculations) the coupling to vibrations as well as the effects of static disorder, the details are given in section II of the SI.

Quantum yield: in the simplest case of the homogeneous aggregate at low temperature we estimated the fluorescence quantum yield as the ratio between the radiative relaxation rate of the lowest optically active excited state and the sum of its radiative relaxation rate, nonradiative relaxation rate, and downward scattering rates to dark (non-emissive) states; we neglected upward scattering processes as they do not play a significant role at low temperature. For the more realistic case of disordered aggregates, we calculated the quantum yield as $Q Y=\left\langle\sum_{k} \frac{\gamma_{k}^{r a d}}{\gamma_{k}^{r a d}+\gamma_{k}^{\text {non-rad }}} P_{k}^{s S}\right\rangle$, where $\gamma_{k}^{r a d}, \gamma_{k}^{\text {non-rad }}$, and $P_{k}^{s s}$ are the radiative rate, the nonradiative rate, and the steady-state population of the state $k$ created by a c.w. pump laser, respectively; the latter were obtained using the PME 
approach. The brackets denote averaging over disorder realizations.

\section{Results and discussion}

\section{III.1 Temperature dependence of absorption and fluorescence linewidths: the role of disorder}

Figure 2a shows the absorption and fluorescence spectra of TPPS4 aggregates and monomers in solutions at room temperature, which are consistent with previous spectroscopic studies. ${ }^{31,34}$. The monomer has two prominent absorption bands: the B band at $436 \mathrm{~nm}(2.85 \mathrm{eV})$, and the $\mathrm{Q}$ band at $647 \mathrm{~nm}(1.92 \mathrm{eV})$. The $\mathrm{B}$ band originates from the degenerate $B_{x}$ and $B_{y}$ transitions; the $\mathrm{Q}$ band consist of two peaks, which originate from $Q_{x, y}(0-1)$ and $Q_{x, y}(0-0)$ transitions, respectively. ${ }^{31,34}$ Upon aggregation, the $B$ band is split into a very narrow red-shifted peak at $490 \mathrm{~nm}(2.53 \mathrm{eV})$ and a wider peak at $423 \mathrm{~nm}(2.93 \mathrm{eV})$; these two peaks originate from different B-band excitons and, as typical for the cylindrical aggregates ${ }^{51}$, they have different polarizations: the low-energy component is polarized parallel to the cylinder axis, while the high-energy component is polarized perpendicular to the aggregate axis. ${ }^{31,34}$

The $Q$ band is also red-shifted upon aggregation by about $0.17 \mathrm{eV}$ to $707 \mathrm{~nm}$ and $675 \mathrm{~nm}$ (also see energy diagram in Figure 4 below). Contrary to the case of the TPPS4 monomer, the second peak in the aggregate's Q-band absorption, at $675 \mathrm{~nm}$, is not a vibronic replica of the main peak at $707 \mathrm{~nm}$, but a different excitonic state. ${ }^{34}$ As in the case of the B-band, these two excitons have different polarizations: the transition dipole of the lowest-energy one is parallel, while the higher energy one is perpendicular to the cylinder axis. ${ }^{31}$ The first vibronic replicas of both aforementioned excitonic states give 
rise to the third peak, at around $630 \mathrm{~nm}$ with no clear polarization.

(a)
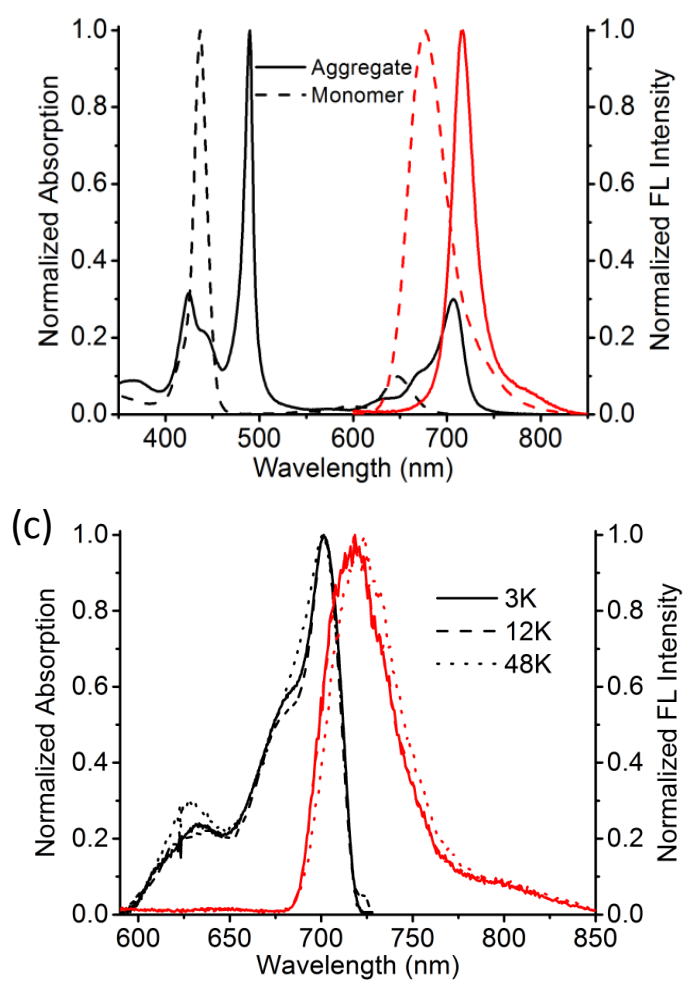

(b)

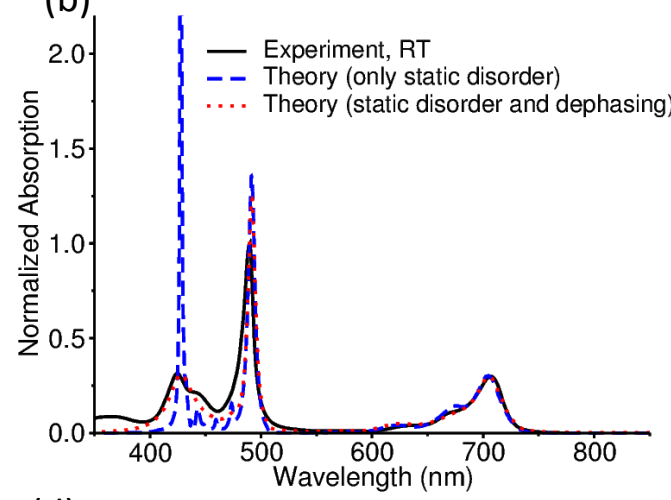

(d)

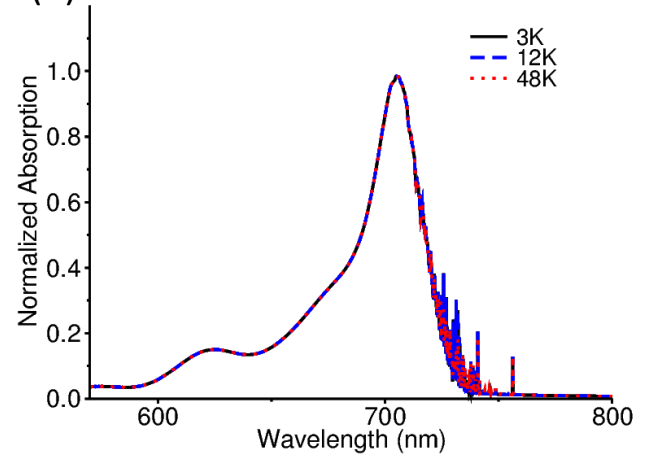

Figure 2 (a) Normalized UV-vis absorption spectra (left) of aggregate and monomer and normalized fluorescence spectra (right, $\lambda_{\mathrm{ex}}=488 \mathrm{~nm}$ for aggregate and $437 \mathrm{~nm}$ for monomer) at room temperature. (b) Simulated absorption spectrum with and without dephasing in comparison to experiment. The model parameters used to obtain the simulated spectra are presented in the text. (c) Measured absorption (black curves) and fluorescence (red curves) spectra in the Q band region for the aggregate at three temperatures: $3 \mathrm{~K}, 12 \mathrm{~K}$, and $48 \mathrm{~K}$. (d) Simulated absorption spectrum in the $\mathrm{Q}$ band region for the aggregate at three different temperatures, using the same model parameters as in Figure 2(b); note that the calculated spectra are almost identical, so the lines overlap to a large extent.

The red-shifted narrow absorption band is the optical signature of the formation of $\mathbf{J}$ aggregates, in which the collective excitonic states (Frenkel excitons) are shared by a number of TPPS4 monomers. ${ }^{17,31,34}$ It has been demonstrated previously that a Frenkel exciton model accounting for four transitions per molecule $\left(B_{x}, B_{y}, Q_{x}\right.$, and $\left.Q_{y}\right)$ provides a good reproduction of the energies and the oscillator strengths of the excited states probed 
in the linear spectroscopy over the entire visible range, ${ }^{31,34}$ however, this model was incapable of correctly reproducing the linewidths. Here, we simulated the absorption linewidths using the Pauli Master Equation approach for inhomogeneous aggregates to account for both the disorder and dephasing (lifetime) contribution to these widths and, for the set of model parameters presented below, good agreement was achieved between theory and experiment, as shown in Figure 2b.

We performed temperature dependent absorption and photoluminescence measurements, the results of which are presented in Figure 2c. The spectra for the monomers are given in Figure S2 of the supporting information. Notably, little temperature dependence was observed in either the absorption or the fluorescence linewidths for the aggregates. In contrast, the fluorescence linewidth of the monomer decreases as temperature decreases (Figure S2).

To understand the temperature dependence of the absorption linewidth, we simulated the temperature dependent absorption spectra as shown in Figure $2 \mathrm{~d}$. The simulations were performed using the methods described in Section II.2, with the one-particle basis set truncated at the 2-phonon states for the Q band and the 0-phonon states for the B band. Temperature enters our model via the Bose-Einstein occupation numbers of the bath modes that govern the values of the scattering rates as shown in Eq. (S3) in SI, and thereby the dephasing rates.

We used a model parameterization based mostly on the experimental absorption spectra of the TPPS4 monomer, which is an extension of the one used in the previous 
work on homogeneous TPPS4 aggregates ${ }^{34}$. Specifically, the mean vertical excitation energies of the TPPS4 monomer were taken as $1.87 \mathrm{eV}$ and $2.85 \mathrm{eV}$ for the $\mathrm{Q}$ and B-band states, respectively, with corresponding transition dipole moments of 4.4 Debye and 11.3 Debye. The transition dipoles are the same as in the previous work, ${ }^{31,34}$ while the excitation energies were altered slightly to accommodate shifts resulting from the more detailed treatment of disorder in the current work and the use of a different solvent in the experiments. As previously, a vibrational quantum of $0.158 \mathrm{eV}$ was used for the high-frequency mode and the Huang-Rhys factors were taken as $\lambda_{Q_{x}}^{2}=\lambda_{Q_{y}}^{2}=0.21$ and $\lambda_{B_{x}}^{2}=\lambda_{B_{y}}^{2}=0$ (see Eq.(S1) in SI). The resonance interactions $J_{n j, m l}$ (Eq.(S1)) were treated as Coulomb interactions between transition dipole moments using the extended dipole approximation, in which the transition dipole moments are represented as pairs of positive and negative charges: the separation between the charges was taken as $0.5 \mathrm{~nm}$, which roughly corresponds to the diameter of a porphyrin ring, and values of $0.182 e$ and $0.470 e$ were used to reproduce the values of the transition dipoles of the $\mathrm{Q}$ and $\mathrm{B}$ transitions, respectively.

The new parameters that needed to be determined for this work were the standard deviation $\sigma$ of the Gaussian distribution of the random static energy shifts $\Delta_{n j}$, as well as the scattering amplitude $W_{0}$ and the spectral density cut-off $\hbar \omega_{C}$ used in the Pauli Master Equation approach (see section II of the SI for definitions of these quantities). These three parameters were determined by fitting to the experimental linewidths of the room-temperature absorption spectrum of the TPPS4 nanotubes over the whole visible 
range, thus for both the $\mathrm{Q}$ and the $\mathrm{B}$ bands at the same time. The best overall fit was obtained for $\sigma=0.045 \mathrm{eV}, W_{0}=3.94 \mathrm{eV}$, and $\hbar \omega_{C}=0.07 \mathrm{eV}$. The values of $W_{0}$ and $\hbar \omega_{C}$ are to some extent related to each other: a good fit to the experimental data still can be obtained by lowering (or increasing) $W_{0}$ by up to $15 \%$ and simultaneous increasing (or lowering) $\hbar \omega_{C}$. On the other hand, a change in $\sigma$ by about $10 \%$ makes the fit much worse.

The simulated positions and widths of the absorption bands of the aggregates (Figure 2d) show little temperature dependence, which is in excellent agreement with experiment (Figure 2c). The explanation is as follows: Scattering by both static disorder and phonons contributes to the exciton linewidth. The former contribution is temperature independent. Phonon scattering may decrease the exciton coherence size below the exciton localization size imposed by static disorder and then broadens the linewidth with growing temperature. As can be seen from Eq. (S3) in the SI, the scattering by phonons has both a temperature-dependent and a temperature-independent contribution. The latter one results from the downward relaxation within the exciton band accompanied by the spontaneous emission of one phonon to the bath. For the states from the middle and top of the band this temperature-independent contribution can be significant even at low temperatures, while it is negligible for the excitons close to the band bottom. In effect, even when the linewidths are dominated by phonon scattering, it is possible that no temperature dependence is observed if the optically dominant states are not located at the band bottom and the temperature-independent contribution to the linewidths is more 
significant than the temperature dependent one. This is exactly the case for the TPPS 4 tubes studied here; because of the cylindrical geometry they are neither precisely J- nor $\mathrm{H}$-aggregates (in the sense that the optically allowed states would occur exactly at the exciton band edges). In the absence of disorder the lowest-energy optically allowed state of the TPPS4 tube is located $0.020 \mathrm{eV}$ above the band bottom (see also the density of states for the homogeneous aggregate in Figure S6 in SI). We note that also for perylene bisimide aggregates the absorption linewidth was observed to exhibit a very weak temperature dependence ${ }^{52}$, which in that case was attributed to a very large level of static disorder, so that inhomogeneous broadening dominates over the homogeneous broadening. ${ }^{52}$

Theoretical modeling aids the interpretation here, as it allows us to disentangle the effects of static disorder and scattering by phonons from each other, by comparing the results of separate simulations that take into account only static disorder with full simulations that account for both effects (see Figure $2 b$ ). We conclude that for the lowest exciton transitions ( $\mathrm{Q}$ band), the absorption linewidth is indeed dominated by static disorder and lifetime broadening by phonon scattering plays a relatively insignificant role. For the B band, on the other hand, both effects are important: for its lower component the linewidth is dominated by the static disorder, while for the higher component it is dominated by scattering on the phonon bath. Still, we expect no temperature dependence of linewidths within the whole $\mathrm{B}$ band, since for the higher component the temperature-independent scattering to lower energy states accompanied by phonon 
emission turns out to be the dominant contribution to the linewidths even at room temperature.

To conclude this section, it is useful to present data on the exciton delocalization sizes imposed by the static disorder. Using the participation number ${ }^{53}$ as measure of the delocalization size of the states simulated by us, we found over the whole Q-band an average delocalization area of 49 molecules (with a standard deviation of 105), while in the whole B-band we found 1150 molecules (standard deviation 262). For the optically dominant states in the Q-band (those lying within an energy $\sigma$ away from the absorption maximum), the average delocalization area equals 208 molecules (standard deviation 152), while at the absorption maximum of the lowest B band we found 1036 (standard deviation 224). However, as manifested by the large values of the standard deviations, many states, especially those close to the band bottom, are delocalized over only a few molecules.

The degree of delocalization of the optically dominant excitons is strikingly large as compared to values generally reported and expected for linear J-aggregates. In the model TPPS4 nanotubes the width of the excitonic Q band in the absence of disorder is about $0.07 \mathrm{eV}$, while the static disorder strength is $0.045 \mathrm{eV}$. In a linear J aggregate such ratio of disorder and bandwidth would lead to optically active excitons delocalized over only a few molecules. ${ }^{53-54}$ One of the reasons for the increased exciton delocalization is the tubular geometry of the aggregate: in the two-dimensional arrangement of the molecules on the surface of the cylinder, the excitation can delocalize in more directions and thereby 
circumvent relatively large disorder fluctuations that may occur at particular molecules. Moreover, we found that, in spite of the considerable energy difference between the molecular Q and B states, the delocalization in the Q band is strongly enhanced by the mixing with the $\mathrm{B}$ band $^{55}$. If the dipole-dipole coupling between the $\mathrm{Q}$ and $\mathrm{B}$ states of different molecules is switched off in our model calculation, the average Q band delocalization area at the absorption maximum reduces from 208 to 88 molecules.

\section{III.2. Excited state absorption: transitions from one-exciton to two-exciton states}

Figure 3 shows experimental and simulated polarization-dependent transient absorption spectra at $0 \mathrm{ps}$ delay at room temperature. The experimental spectra were obtained with a pump wavelength fixed at $488 \mathrm{~nm}$ and varying the probe wavelength, using a probe polarization parallel or perpendicular to the polarization of the pump pulse. The simulated spectra were obtained using the hard-core boson approach as mentioned in section II.2, restricting to homogeneous aggregates without coupling to vibrations to reduce the computational cost. ${ }^{56}$ This approach accounts for all one-exciton and two-exciton states originating from the $\mathrm{Q}$ and $\mathrm{B}$ bands. The initial state, created by the pump pulse, was taken as the excitation of the lower B band transition, to which the pump pulse was tuned. For the simulated spectra shown in Figure 3, we did not allow any evolution of this initially created state before the probe pulse arrives. Figure S7 shows that the spectra obtained using this assumption do not differ dramatically from those calculated assuming that the exciton populations in the Q and B bands have reached overall thermal equilibrium before the probe pulse arrives. Because the pulse width of 
the pump is $\sim 150 \mathrm{fs}$, on the same order as intraband relaxation time, ${ }^{39}$ the actual experimental condition is in between these two extreme cases.

In the visible-near IR spectral region the experimental spectra seem to be dominated by ground state bleaching of the Q band transitions and a broad excited state absorption band at lower wavelengths, consistent with previous reports ${ }^{37,40}$ on these aggregates. Changing from parallel to perpendicular probe polarization, the ratio of the $675 \mathrm{~nm}$ and the $707 \mathrm{~nm}$ bleaching peaks grows. Though the calculated spectra have a more pronounced structure in the $\mathrm{Q}$ band region than the experimental ones, they do reproduce the bleaching peaks with similar polarization dependence. The simulations fail to reproduce the broad excited state absorption band below $650 \mathrm{~nm}$. We will discuss these various observations in the following.

The polarization dependence is simply understood from the excitation conditions. The lower B band transition excited by the pump pulse at $488 \mathrm{~nm}$ is polarized mainly parallel to the tube's axis. ${ }^{31,34}$ This implies that tubes have a higher probability to be excited the closer their axes are oriented to the pump's polarization direction. In other words, the pump selects an anisotropic distribution of tube orientations. Therefore, when the probe polarization is parallel to that of the pump, it also is parallel to the majority of tubes that have been excited by the pump and hence it will preferentially be sensitive to the $707 \mathrm{~nm}$ peak, as this is polarized parallel to the tube's axis as well. ${ }^{31,34}$ On the other hand, when the probe has polarization perpendicular to the pump, it will preferentially be sensitive to the $675 \mathrm{~nm}$ peak, as this transition is polarized perpendicular to the tube's 
axis. In fact, our measurements confirm the polarization directions of the two main transitions underlying the $\mathrm{Q}$ band in the aggregate, which have been assigned to the $0 \rightarrow 0$ vibronic transition of the lower and upper Bethe components, respectively. ${ }^{34}$

As noted above, the simulated spectra show a more pronounced excited state absorption structure in the $\mathrm{Q}$ band region than the experimental ones: in the simulations both negative bleaching (and simulated emission) peaks are accompanied by a narrow lower-wavelength excited state absorption contribution. This results from the dominant (superradiant) transitions from the B-band's one-exciton states occupied after pumping the system to two-exciton states associated with the mixed Q/B two-exciton band (this is a band that contains superpositions of states in which two molecules are electronically excited; these molecules may (independently) be in either a Q or a B state; also see Figure 4 below); the blue shift relative to the associated bleaching peak originates from the Pauli exclusion: each molecule only can carry one excitation ${ }^{57}$. (For the case of equilibration before probing, the similar narrow excited state absorption peaks in Fig. S7 originate from transitions from both the $\mathrm{B}$ and the $\mathrm{Q}$ band to the mixed $\mathrm{Q} / \mathrm{B}$ two-exciton states.) The fact that these excited state transitions cannot be discerned in the experimental spectra probably is due to the larger linewidths that smoothen the structure as well as vibronic effects. 


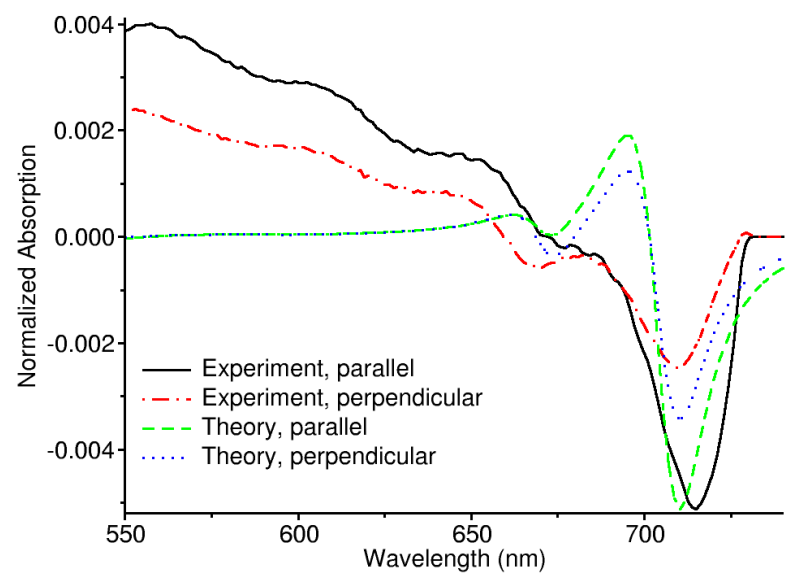

Figure 3. Measured and simulated polarization-dependent transient absorption spectra of TPPS4 aggregates at $0 \mathrm{ps}$ delay at $298 \mathrm{~K}$. The polarization of the probe was parallel or perpendicular to the pump polarization.

As noted above, the simulated spectra do not reproduce the strong excited state absorption signal at wavelengths ranging from $550 \mathrm{~nm}$ to $650 \mathrm{~nm}$. It turns out hard to unambiguously explain this experimental signal. As demonstrated in Figure S7, the failure to describe it within our model is not an artifact of our assumption that the pump B band state does not relax before the probe pulse arrives. We also note that degradation of the samples as cause for the strong excited state absorption contributions, changing the exciton level structure, can be ruled out. We made sure that the samples did not degrade during the pump-probe measurements by checking the absorption spectra before and after the measurements. We did not observe measurable differences in the spectra before and after the pump-probe measurements.

It should be noted, however, that a strong and broad excited state absorption signal also is present in the transient absorption of the TPPS4 monomer (Figure S3 in SI). Based on the wavelength range over which this signal prevails, we ascribe it to transitions from the Q band states in the TPPS4 monomer to higher excited states " $\mathrm{X}$ " lying above the B 
band (see Figure 4 for the proposed energy level diagram). In the aggregate this will also lead to additional excited state absorption contributions from the Q or the B band states to the manifold of $\mathrm{X}$ states (or rather, since we estimate the single-exciton band originating from the molecular $\mathrm{X}$ states to lie in an energetic range that is similar to that of the mixed Q/B two-exciton band (Figure 4), to the manifold of mixed states formed from the $\mathrm{Q} / \mathrm{B}$ two-exciton states and the $\mathrm{X}$ one-exciton band). Whether or not this is the explanation to the observed discrepancy between theory and experiment is uncertain, because the transition from the $\mathrm{Q}$ or $\mathrm{B}$ one-exciton band to the one-exciton $\mathrm{X}$ states cannot be superradiantly enhanced, which makes its contribution to the spectrum much weaker than superradiant transitions dominating the spectrum. Only a complete calculation of the pump-probe spectrum, including the $\mathrm{Q}, \mathrm{B}$, and $\mathrm{X}$ states as well as disorder and vibronic effects, may reveal to what extent cancellation of close lying positive and negative peaks in the low-energy region of the pump-probe spectrum may effectively lower the intensity there, so that the $\mathrm{X}$ state indeed may explain the observed high-energy excited state absorption. Such a complete calculation computationally is too demanding to be carried out. 


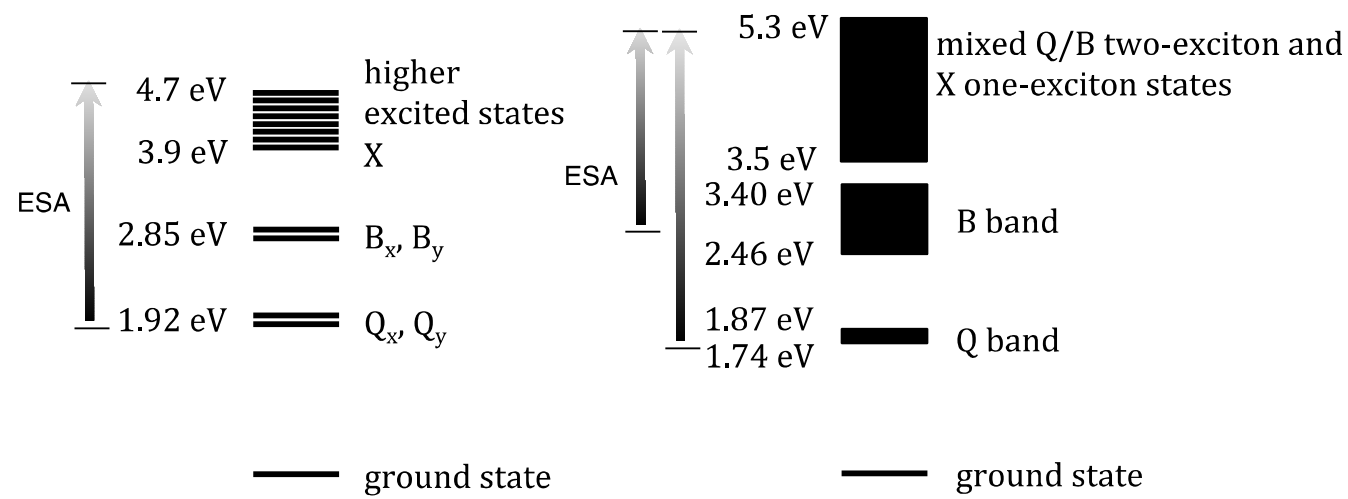

Figure 4. Energy level diagram of the TPPS4 monomer (left) and aggregate (right). ESA: excited state absorption, the gradient arrows indicate that their end points in principle may be anywhere in the receiving band.

\section{III.3 Temperature dependent fluorescence lifetime and dynamic Stokes shift}

The spectrally integrated fluorescence decays at different temperatures for the monomers and the aggregates are shown in Figure 5. Excitation wavelengths for the monomer and the aggregate were $437 \mathrm{~nm}$ and $488 \mathrm{~nm}$, corresponding to their respective B band resonances. In these experiments the pump intensity was kept below $4 \mu \mathrm{J} / \mathrm{cm}^{2}$ corresponding to an excitation density of around one exciton per 1000 TPPS4 molecules. The monomer exhibits single exponential fluorescence decay at all temperatures with a life time that increases from $4.1 \mathrm{~ns}$ at room temperature to $7.8 \mathrm{~ns}$ at $3 \mathrm{~K}$ (Figure 5).

In contrast, the fluorescence decay of the aggregate is highly nonexponential. A tri-exponential decay function convoluted with a Gaussian response function is utilized to fit this fluorescence decay. The decay constants along with the corresponding weights as a function of temperature are plotted in Figure $5 \mathrm{c}$ and summarized in Table S1. The three decay constants are on the order of tens of picoseconds, hundreds of picoseconds, and a 
few nanoseconds, respectively. All three lifetimes increase as temperature decreases (Figure 5c). We also measured the fluorescence quantum yield (QY) at various temperatures and the results are summarized in Figure S4 in the SI. At $3 \mathrm{~K}$ the monomer has a QY of 0.04 , whereas that of the aggregates is 20 fold lower at $\sim 0.002$. Such low QYs indicate that nonradiative pathways dominate energy relaxation in both monomers and aggregates. The much faster and nonexponential nature of the fluorescence decay of the aggregate suggests that an inhomogeneous distribution of states, most likely due to disorder, is involved ${ }^{58}$. Nonexponential decays were also observed in tubular cyanine aggregates. ${ }^{58}$ Further discussion about the radiative and nonradiative pathways is provided in section III.4.

Figure 6 shows the spectrally resolved fluorescence lifetime spectra of aggregate and monomer measured at $3 \mathrm{~K}$. The data at room temperature are shown in Figure S5. Spectra at different delay times were obtained by integrating the streak camera results over an interval of $0.2 \mathrm{~ns}$ and decay curves at different wavelengths were obtained by integrating over a spectral window of $10 \mathrm{~nm}$. At room temperature, the fluorescence lifetime has no spectral dependence for either the aggregate or the monomer (Figure S5), implying that for both systems thermal equilibrium is reached in the excited state manifold within this lifetime. But at very low temperature, such as $3 \mathrm{~K}$, the aggregate fluorescence spectra exhibit a distinctive dynamic Stokes shift (Figure 6). They red-shift with increasing time delay, indicating that the exciton populations at the higher energy side decay faster than those at the lower energy side. Such a dynamic Stokes shift is 
absent for the monomer at all temperatures.
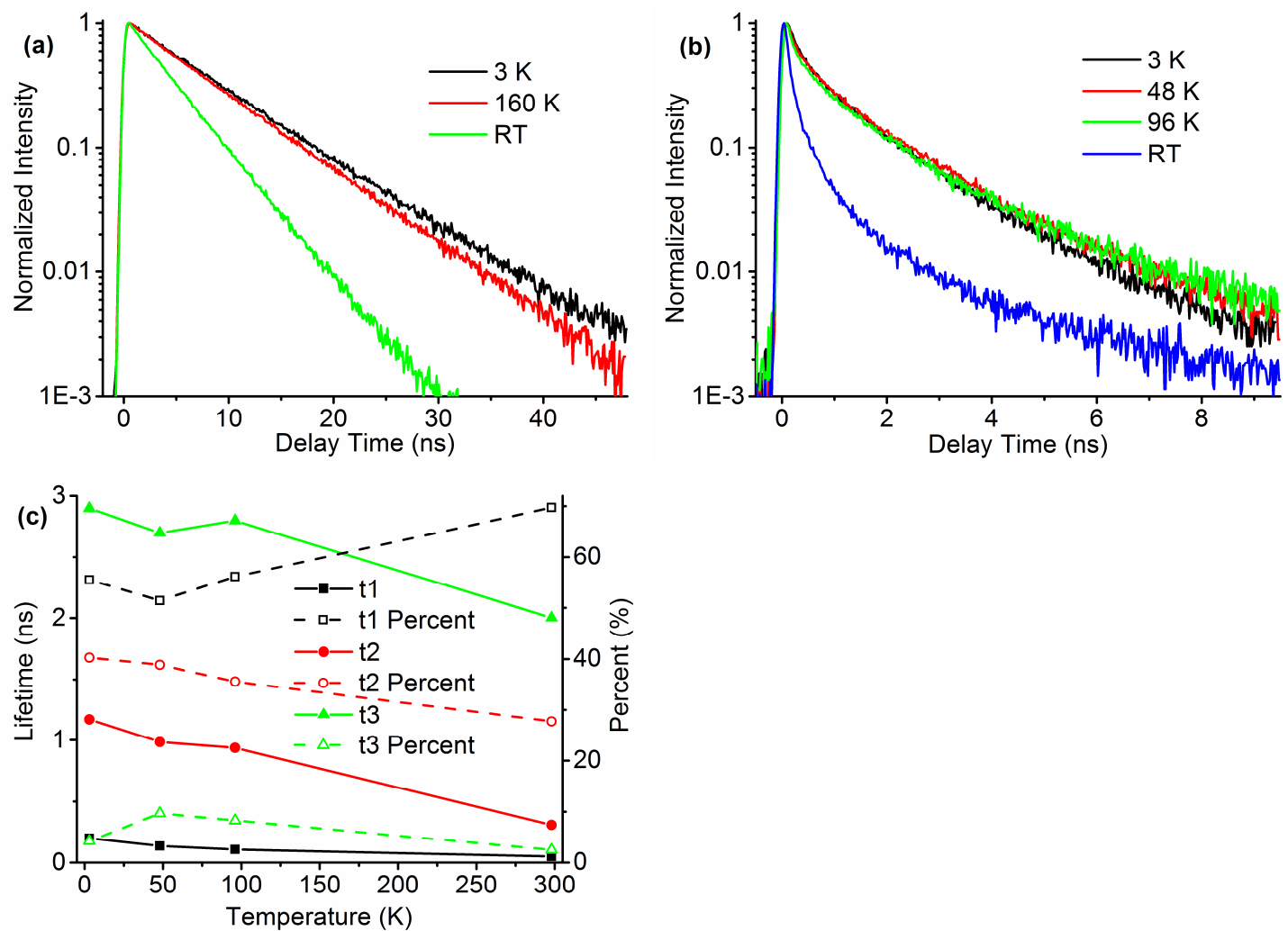

Figure 5. Fluorescence dynamics of monomer (a) and aggregate (b) measured at different temperatures; (c) Fluorescence decay constants (left axis) of aggregates and their contribution (right axis) at different temperatures.

Similar dynamic Stokes shifts have been observed in tubular aggregates formed from cyanine dyes and were attributed to the relaxation within the inhomogeneously broadened density of states while fluorescence decay occurs. ${ }^{58}$ We ascribe the dynamical Stokes shift observed here to the same origin, and this provides yet another indication of significant static disorder in the TPPS4 aggregates. For high temperature, the intraband scattering rates $W_{k l}$ (Eq. (S3)) are large and the exciton system equilibrates prior to fluorescence decay; no dynamic Stokes shift is observed then. At low temperature, the intraband relaxation slows down, which may result in down-ward relaxation occurring on 
the same time scale as fluorescence emission, thus giving rise to a dynamic Stokes shift. Not reaching thermal equilibrium at very low temperatures even may result in a nonmonotonous temperature dependence of the Stokes shift of the steady state fluorescence spectrum. ${ }^{58-60}$
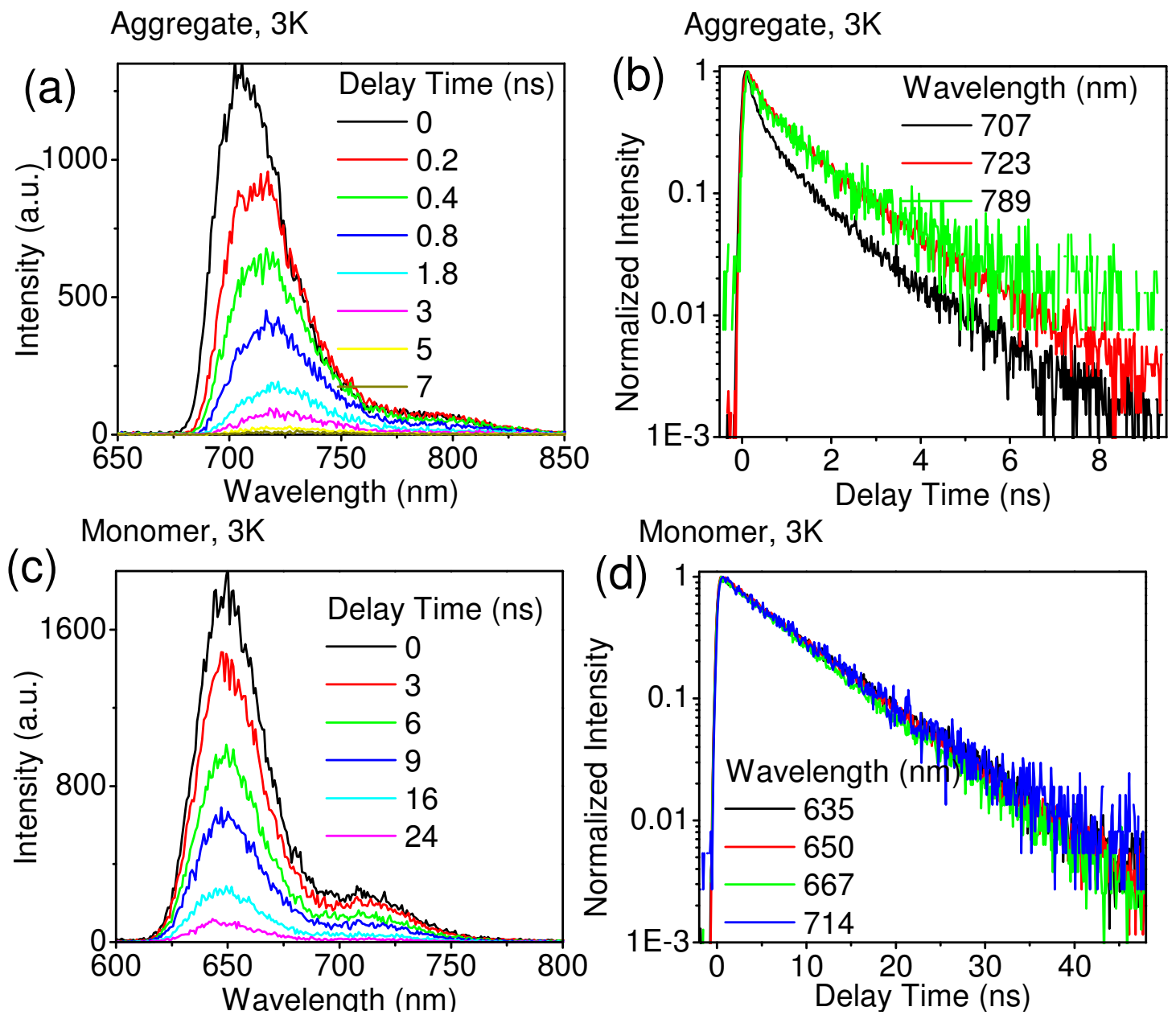

Monomer, 3K

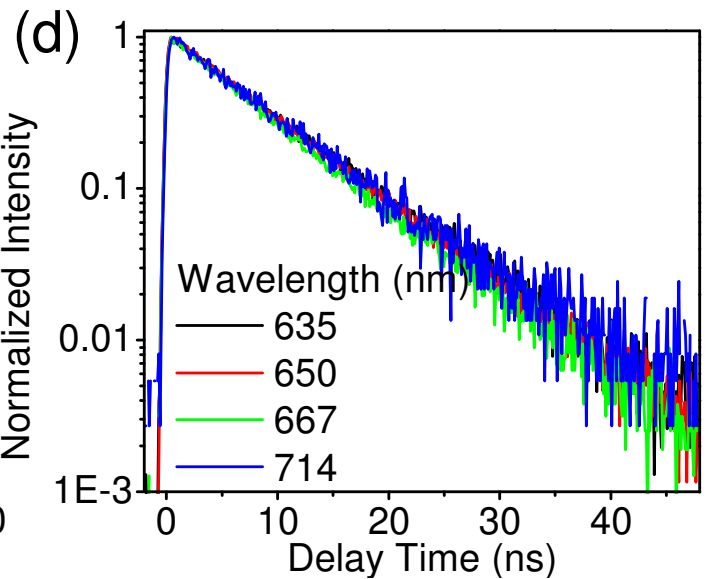

Figure 6. Transient fluorescence spectra and spectrally resolved fluorescence decay traces of aggregate $(a, b)$ and monomer $(c, d)$ measured at $3 \mathrm{~K}$.

\section{III.4. Radiative and nonradiative processes}

In this section we discuss the role of disorder and the nature of the radiative and nonradiative relaxation pathways of the Frenkel excitons in the TPPS4 tubular aggregates. 
In order to elucidate the lifetimes of the excited states, global analysis has been used to resolve the corresponding excited state spectral features and to determine their lifetimes. ${ }^{61}$ Note that the time window of the transient absorption measurements is $\sim 1 \mathrm{~ns}$, so that the slowest decay component of a few nanoseconds is not fully resolved and can be considered to be non-decaying. The spectral surfaces are fitted using two exponentials plus a non-decaying (> $1 \mathrm{~ns})$ component.

The resulting decay-associated spectra (DAS) are shown in Figure 7 (details in the SI). Both the recovery of the ground state bleach and the relaxation of the excited state absorption are highly nonexponential at all temperatures with decay time constants ranging from a few picoseconds to a few nanoseconds. The fastest decaying DAS, which can be associated with a lifetime of $1.2 \mathrm{ps}$ at $4 \mathrm{~K}$ and $4.2 \mathrm{ps}$ at room temperature $(293 \mathrm{~K})$, reveal spectral features of both the bleaching of the two lowest exciton states and a broad excited state absorption, reminiscent of the excited state absorption discussed in section III.2. At $3 \mathrm{~K}$, the DAS at $1.2 \mathrm{ps}$ is red-shifted compared to the slower decaying DAS components, most likely due to the contribution of stimulated emission. The slowest-decay (i.e., non-decaying) DAS consists of mostly ground state bleach as is suggested by the fact that it has almost no excited state absorption features. 

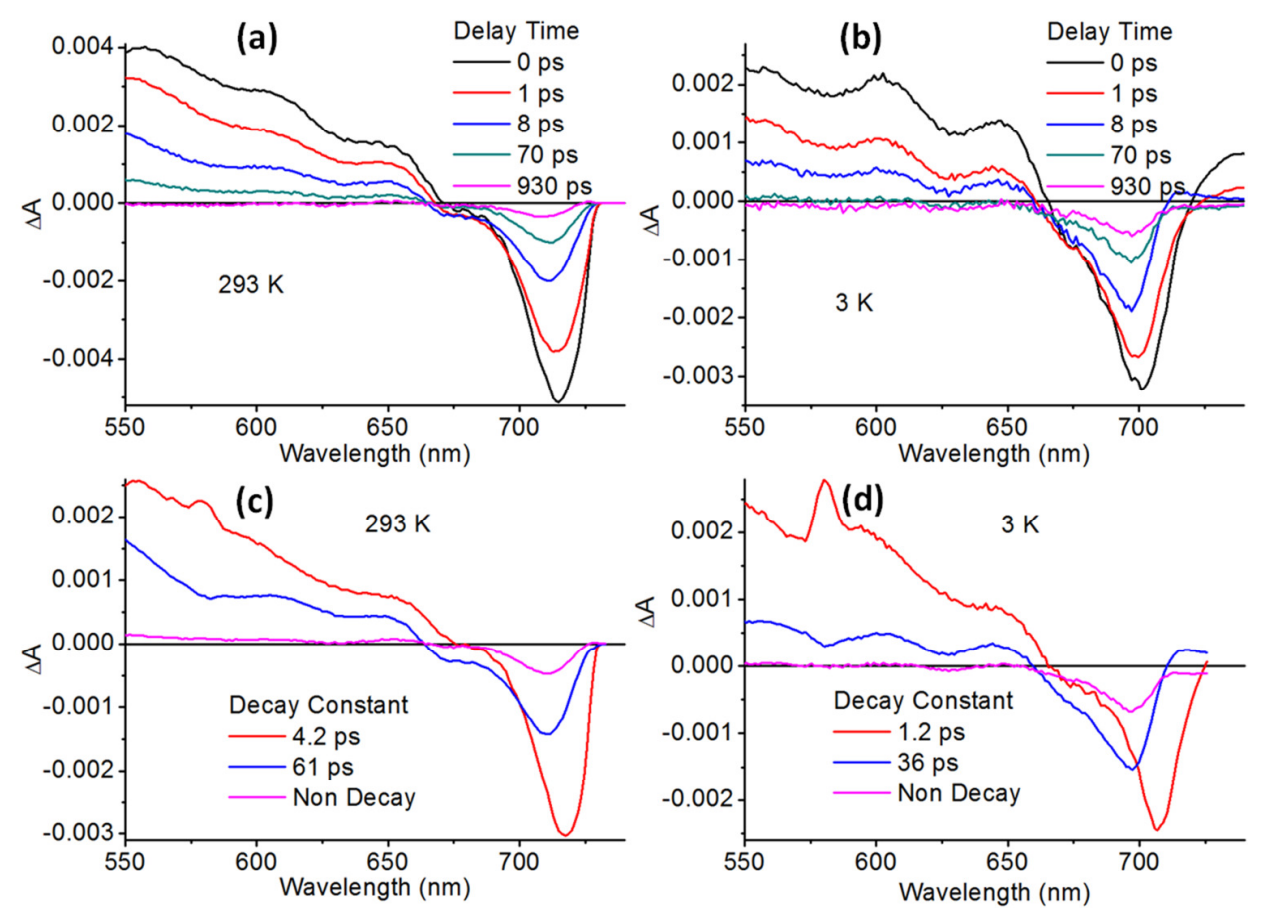

Figure 7. Temperature dependent transient absorption spectroscopy of aggregates at RT (a) and $3 \mathrm{~K}$ (b). The excitation wavelength was $488 \mathrm{~nm}$ and the polarization of the probe beam was parallel to the pump beam. The decay-associated spectra (DAS) of aggregates at RT (c) and $3 \mathrm{~K}$ (d) obtained by global analysis.

The low QY (0.002 at $4 \mathrm{~K})$ indicates that nonradiative pathways dominate energy relaxation in the aggregates. Figure 8 compares the relaxation of the excited state absorption and the recovery of the ground state bleach with the fluorescence decay at $4 \mathrm{~K}$. The excited state absorption decay lacks the nanosecond decay component compared to the ground state recovery, consistent with the DAS results, which can be explained by the fact that the state where the excited state absorption initiates from relaxes into a different excited state (for instance, a dark state due to defects or disorder, or an excimer state) before the ground state fully recovers on the nanosecond timescale. The ground state recovery has an additional fast component on the order of $1 \mathrm{ps}$ compared to the fluorescence decay. One possible explanation for this 1 ps decay is exciton-exciton 
annihilation, because the pump intensity for the transient absorption measurements creates about one exciton /100 molecules, compared to one exciton /1000 molecules for the fluorescence lifetime measurements. As shown in Figure S8 of the SI, the amplitude of the 1 ps decay component in the pump probe measurements increases as the pump intensity increases, indicating that, indeed, exciton-exciton annihilation plays a role. We do not observe a significant exciton-exciton annihilation effect in the intensity-dependent PL measurements, not even for intensities that result in one exciton per 250 molecules (Figure S8). Considering that the Q band exciton is delocalized over $\sim 200$ molecules, the last observation suggests that exciton localization probably occurs prior to exciton motion, although our experiments do not directly provide evidence for such ultrafast localization. Another possible explanation for the $1 \mathrm{ps}$ component is that dark states with fast nonradiative relaxation are involved in the ground state recovery process. This is because fluorescence decay reflects only the population of the emitting states while ground state recovery tracks the population of all excited states (bright and dark).

As discussed in section III.1, static disorder dominates the absorption linewidth for the lowest exciton energy level. The nonexponential exciton relaxation and the dynamic Stokes shift observed in the aggregates are both manifestations of an inhomogeneously broadened exciton density of states. The next step is to understand the lowering of the QY in the aggregate as compared to the monomer, as that should provide us with deeper insight into the exciton dynamics. In a first attempt to model the aggregate's QY, we used methods described in Section II.2. Here, we used the low-temperature (3K) radiative rate 
of the monomer $\gamma_{0}^{r a d}$ of $0.0051 \mathrm{~ns}^{-1}$, based on its QY of 0.04 and the $7.8 \mathrm{~ns}$ fluorescence lifetime. Furthermore, we assumed that the nonradiative relaxation rate to the ground state is the same for all the aggregate's excited states and equal to the monomer's nonradiative relaxation rate $\gamma_{0}^{\text {non-rad }}$ of $0.12 \mathrm{~ns}^{-1}$. For the perfectly homogeneous aggregate our model predicts a QY of 0.0013 , which is of the same order of magnitude as the experimental value 0.002 . For the more realistic case of an inhomogeneous aggregate, however, using Eq. (3) we obtain a QY of 0.08, which is almost twice that of the monomer. This observation suggests that the agreement between theory and experiment obtained for the homogeneous aggregate is a mere coincidence.

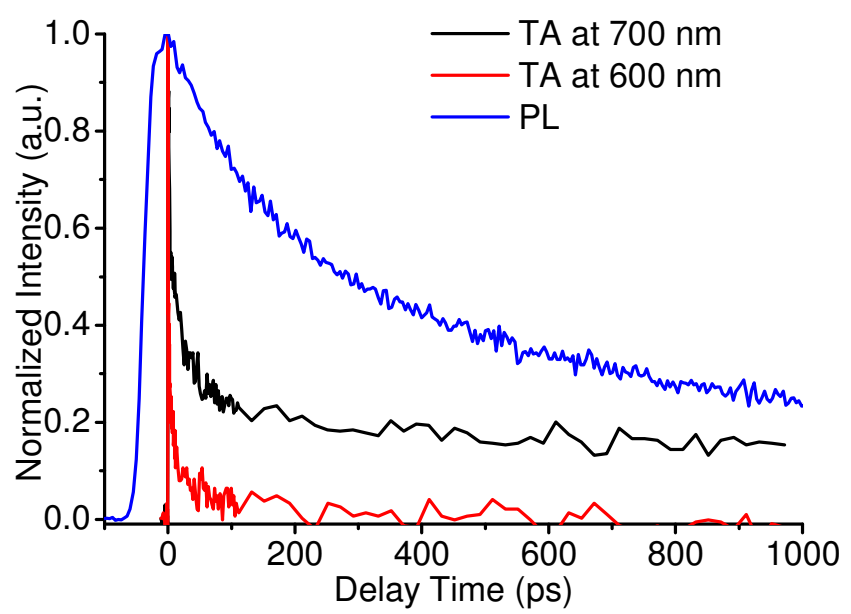

Figure 8. Transient absorption (TA) decay dynamics taken at the excited state absorption $(600 \mathrm{~nm})$ as well as at the ground state bleach $(700 \mathrm{~nm})$ compared with fluorescence (PL) decay at $4 \mathrm{~K}$. The excitation wavelengths were all at the B band resonance at $488 \mathrm{~nm}$.

The strongly reduced QY for a homogenous aggregate can be understood from its density of states (Figure S6 in the SI): in the absence of disorder, the lowest optically allowed (emissive) exciton state is not at the bottom of the Q band and phonon-induced 
intraband relaxation from this state to the lower-energy symmetry-forbidden dark exciton states is more effective than its radiative relaxation. This explains the strong reduction of the predicted quantum yield. However, once disorder is introduced, the cylindrical symmetry is broken and the low-energy dark states become optically allowed; the aggregate's predicted QY then essentially becomes twice that of the monomer. This doubling of the QY in our simulations results from the fact, that at $3 \mathrm{~K}$ the fluorescence originates from the strongly localized states close to the band bottom, which carry about twice the oscillator strength of the monomer (at such low temperature the population of the strongly delocalized states at $0.02 \mathrm{eV}$ above the band bottom is negligible).

We conclude that phonon-induced scattering within an inhomogeneous exciton band resulting from Gaussian diagonal disorder does not explain the lowering of the QY upon aggregation. Thus, another mechanism is responsible, not included in our model. So far we disregarded off-diagonal disorder as well as structural defects. Off-diagonal disorder refers to variations in the dipolar coupling between adjacent molecules and can be induced by fluctuations in the local configuration ${ }^{53}$. Structural defects can lead to traps or dark states that contribute to nonradiative processes. Rapid relaxation into dark states $(\sim \mathrm{a}$ few ps) can explain the transient absorption and fluorescence dynamics observed (section III.3) and is most likely responsible for the observed low QY in the aggregates. The exact nature of the off-diagonal disorder and dark states is beyond the scope of this paper and will be the topic of future investigations.

\section{Conclusions}


In conclusion, we reported a comprehensive account of the optical properties of the Frenkel excitons in self-assembled porphyrin tubular aggregates. Such an aggregate can serve as a model system to understand energy transport in natural photosynthetic antennae. We investigated both linear and nonlinear exciton absorption, relaxation pathways, and the role of disorder using combined ultrafast spectroscopy and stochastic exciton modeling methods. In spite of the occurrence of site disorder of $0.045 \mathrm{eV}$, strong intermolecular interactions lead to exciton delocalization across, on average, about 208 molecules in the lower -optically dominant- part of the aggregate's Q band, while at the lowest absorption peak of the B band an average size of even 1036 molecules is found. The delocalization in the $\mathrm{Q}$ band is enhanced by mixing with the $\mathrm{B}$ band.

The absorption and fluorescence linewidths show little temperature dependence and in the low energy range were found to be dominated by static disorder. The temperature dependence of the linear absorption spectra have been simulated using the Pauli Master Equation approach assuming scattering on acoustic phonons in the host matrix; this provided excellent agreement with the observed data. We attributed excited state absorption to transitions from one-exciton states to $\mathrm{Q} / \mathrm{B}$ two-exciton states, possibly mixed with one-exciton states originating from an even higher lying molecular excited state $\mathrm{X}$. Both the transient absorption of and the fluorescence from the exciton of the tubular aggregates are marked by non-exponential decays ranging from a few picoseconds to a few nanoseconds and were ascribed to complex relaxation mechanisms dominated by disorder and dark states. Theoretical modeling of the fluorescence QY 
suggests that nonradiative pathways induced by disorder and structural defects (dark states) dominate the nonradiative relaxation of excitons in the tubular aggregates. We conclude that although the delocalized Frenkel excitons in these aggregates could potentially facilitate energy transfer, fast relaxation induced by defects and disorder presents a major limitation for exciton transport over large distances. 
Acknowledgement: Y. Wan, Z. Guo, S. Fong, and L. Huang were supported by the Division of Chemical Sciences, Geosciences and Biosciences, Office of Basic Energy Sciences of the US Department of Energy through grant DE-FC02-04ER15533. Use of the Center for Nanoscale Materials was supported by the U. S. Department of Energy, Office of Science, Office of Basic Energy Sciences, under Contract No. DE-AC02-06CH11357. This publication is contribution No. NDRL 5030 from the Notre Dame Radiation Laboratory. A. Stradomska acknowledges the Netherlands Organisation for Scientific Research for support through a VENI grant.

\section{Supporting Information Available}

Supporting figures and tables as well as additional details on theoretical modeling and global analysis of the transient absorption spectra. This information is available free of charge via the internet at http://pubs.acs.org.

\section{References:}

TOC
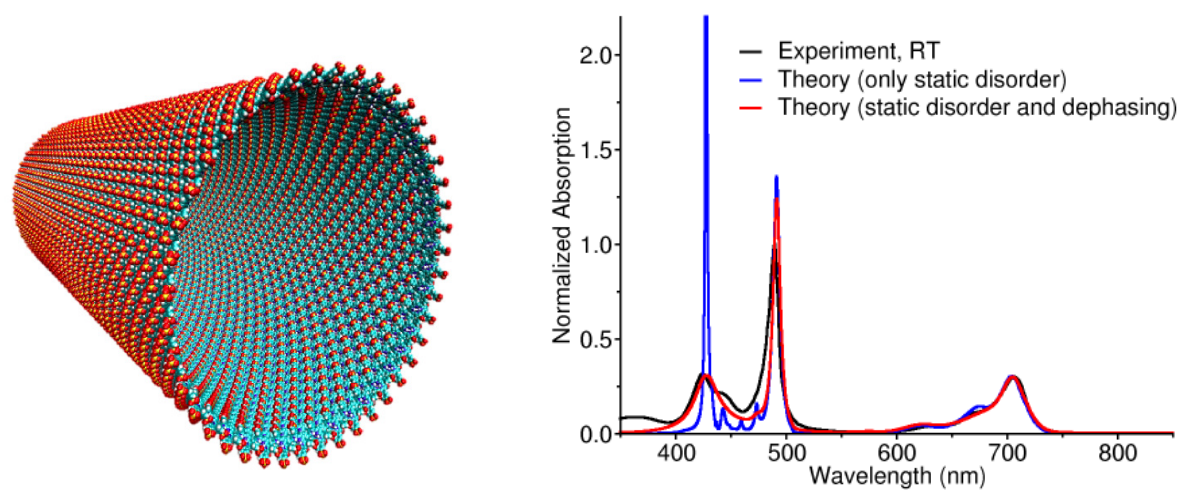
(1) Bahatyrova, S.; Frese, R. N.; Siebert, C. A.; Olsen, J. D.; Van Der Werf, K. O.; Van Grondelle, R.; Niederman, R. A.; Bullough, P. A.; Otto, C.; Hunter, C. N., The native architecture of a photosynthetic membrane. Nature 2004, 430, 1058-1062.

(2) Scholes, G. D.; Fleming, G. R.; Olaya-Castro, A.; Van Grondelle, R., Lessons from nature about solar light harvesting. Nature Chemistry 2011, 3, 763-774.

(3) Blankenship, R. E.; Tiede, D. M.; Barber, J.; Brudvig, G. W.; Fleming, G.; Ghirardi, M.; Gunner, M. R.; Junge, W.; Kramer, D. M.; Melis, A.; Moore, T. A.; Moser, C. C.; Nocera, D. G.; Nozik, A. J.; Ort, D. R.; Parson, W. W.; Prince, R. C.; Sayre, R. T., Comparing photosynthetic and photovoltaic efficiencies and recognizing the potential for improvement. Science 2011, 332, 805-809.

(4) Fleming, G. R.; Schlau-Cohen, G. S.; Amarnath, K.; Zaks, J., Design principles of photosynthetic light-harvesting. Faraday Discussions 2012, 155, 27-41.

(5) Balaban, T. S., Tailoring porphyrins and chlorins for self-assembly in biomimetic artificial antenna systems. Accounts of chemical research 2005, 38, 612-23.

(6) Medforth, C. J.; Wang, Z.; Martin, K. E.; Song, Y.; Jacobsen, J. L.; Shelnutt, J. A., Self-assembled porphyrin nanostructures. Chemical Communications 2009, 7261-7277.

(7) Marciniak, H.; Li, X.-Q.; Würthner, F.; Lochbrunner, S., One-dimensional exciton diffusion in perylene bisimide aggregates. Journal Of Physical Chemistry A 2011, 115, 648-654.

(8) Walker, B. J.; Dorn, A.; Bulović, V.; Bawendi, M. G., Color-Selective Photocurrent Enhancement in Coupled J-Aggregate/Nanowires Formed in Solution. Nano Lett 2011, 11, 2655-2659.

(9) McHale, J. L., Hierarchal Light-Harvesting Aggregates and Their Potential for Solar Energy Applications. Journal Of Physical Chemistry Letters 2012, 3, 587-597.

(10)Sengupta, S.; Würthner, F., Chlorophyll J-Aggregates: From Bioinspired Dye Stacks to Nanotubes, Liquid Crystals, and Biosupramolecular Electronics. Accounts of chemical research 2013, 46, 2498-2512.

(11)Fidder, H.; Knoester, J.; Wiersma, D. A., Superradiant emission and optical dephasing in J-aggregates. Chemical Physics Letters 1990, 171, 529-536.

(12)Spano, F. C.; Kuklinski, J. R.; Mukamel, S., Cooperative radiative dynamics in molecular aggregates. The Journal of Chemical Physics 1991, 94, 7534-7544.

(13)Scholes, G.; Jordanides, X.; Fleming, G., Adapting the Forster theory of energy transfer for modeling dynamics in aggregated molecular assemblies. The journal of physical chemistry B 2001, 105, 1640-1651.

(14)Huijser, A.; Suijkerbuijk, B. M. J. M.; Gebbink, R. J. M. K.; Savenije, T. J.; Siebbeles, L. D. A., Efficient exciton transport in layers of self-assembled porphyrin derivatives. Journal Of The American Chemical Society 2008, 130, 2485-2492.

(15)Lin, H.; Camacho, R.; Tian, Y.; Kaiser, T.; Würthner, F.; Scheblykin, I., Collective Fluorescence Blinking in Linear J-Aggregates Assisted by Long-Distance Exciton 
Migration. Nano Lett 2009, 10, 620-626.

(16)Kasha, M., Energy Transfer Mechanisms and the Molecular Exciton Model for Molecular Aggregates. Radiation research 1963, 20, 55-70.

(17) Spano, F. C., The spectral signatures of Frenkel polarons in H- and J-aggregates. Accounts of chemical research 2010, 43, 429-439.

(18)Roger, C.; Miloslavina, Y.; Brunner, D.; Holzwarth, A. R.; Wurthner, F., Self-assembled zinc chlorin rod antennae powered by peripheral light-harvesting chromophores. J Am Chem Soc 2008, 130, 5929-39.

(19)Nam, Y.; Shin, T.; Park, H.; Magyar, A.; Choi, K.; Fantner, G.; Nelson, K.; Belcher, A., Virus-templated assembly of porphyrins into light-harvesting nanoantennae. Journal Of The American Chemical Society 2010, 132, 1462-1463.

(20)Eisele, D. M.; Cone, C. W.; Bloemsma, E. A.; Vlaming, S. M.; van der Kwaak, C. G. F.; Silbey, R. J.; Bawendi, M. G.; Knoester, J.; Rabe, J. P.; Vanden Bout, D. A., Utilizing redox-chemistry to elucidate the nature of exciton transitions in supramolecular dye nanotubes. Nature Chemistry 2012, 4, 655-662.

(21)Shibata, Y.; Tateishi, S.; Nakabayashi, S.; Itoh, S.; Tamiaki, H., Intensity Borrowing via Excitonic Couplings among Soret and Q yTransitions of Bacteriochlorophylls in the Pigment Aggregates of Chlorosomes, the Light-Harvesting Antennae of Green Sulfur Bacteria. Biochemistry 2010, 49, 7504-7515.

(22)Orf, G. S.; Blankenship, R. E., Chlorosome antenna complexes from green photosynthetic bacteria. Photosynthesis Research 2013.

(23) Ganapathy, S.; Oostergetel, G. T.; Wawrzyniak, P. K.; Reus, M.; Gomez Maqueo Chew, A.; Buda, F.; Boekema, E. J.; Bryant, D. A.; Holzwarth, A. R.; de Groot, H. J. M., Alternating syn-anti bacteriochlorophylls form concentric helical nanotubes in chlorosomes. Proc. Natl. Acad. Sci. U.S.A. 2009, 106, 8525-8530.

(24)Jendrny, M.; Aartsma, T. J.; Kohler, J., Fluorescence Excitation Spectra from Individual Chlorosomes of the Green Sulfur Bacterium Chlorobaculum tepidum. Journal Of Physical Chemistry Letters 2012, 3, 3745-3750.

(25)Schwab, A.; Smith, D.; Rich, C.; Young, E.; Smith, W.; de Paula, J., Porphyrin nanorods. The journal of physical chemistry B 2003, 107, 11339-11345.

(26)Castriciano, M. A.; Romeo, A.; Villari, V.; Micali, N.; Scolaro, L. M., Structural Rearrangements in 5,10,15,20-Tetrakis(4-sulfonatophenyl)porphyrin J-Aggregates under Strongly Acidic Conditions. The journal of physical chemistry B 2003, 107, 8765-8771.

(27)Rotomskis, R.; Augulis, R.; Snitka, V.; Valiokas, R.; Liedberg, B., Hierarchical structure of TPPS4 J-aggregates on substrate revealed by atomic force microscopy. The journal of physical chemistry B 2004, 108, 2833-2838.

(28)Snitka, V.; Rackaitis, M.; Rodaite, R., Assemblies of TPPS4 porphyrin investigated by TEM, SPM and UV-vis spectroscopy. Sensors and Actuators B: Chemical 2005, 109, 159-166.

(29)Friesen, B. A.; Nishida, K. R. A.; McHale, J. L.; Mazur, U., New Nanoscale Insights into the Internal Structure of Tetrakis(4-sulfonatophenyl) Porphyrin Nanorods. Journal 
Of Physical Chemistry C 2009, 113, 1709-1718.

(30)Hollingsworth, J. V.; Richard, A. J.; Vicente, M. G. H.; Russo, P. S., Characterization of the Self-Assembly of meso-Tetra(4-sulfonatophenyl)porphyrin (H 2TPPS 4-) in Aqueous Solutions. Biomacromolecules 2012, 13, 60-72.

(31) Vlaming, S. M.; Augulis, R.; Stuart, M. C. A.; Knoester, J.; van Loosdrecht, P. H. M., Exciton Spectra and the Microscopic Structure of Self-Assembled Porphyrin Nanotubes. The journal of physical chemistry B 2009, 113, 2273-2283.

(32) Kalimuthu, P.; John, S. A., Nanostructured aggregates of meso-tetramesitylporphyrin on solid substrate. Langmuir : the ACS journal of surfaces and colloids 2009, 25, 12414-8.

(33)Short, J. M.; Berriman, J. A.; Kübel, C.; El-Hachemi, Z.; Naubron, J.-V.; Balaban, T. S., Electron Cryo - Microscopy of TPPS4 - 2HCl Tubes Reveals a Helical Organisation Explaining the Origin of their Chirality. Chemphyschem 2013, 14, 3209-3214.

(34)Stradomska, A.; Knoester, J., Shape of the Q band in the absorption spectra of porphyrin nanotubes: Vibronic coupling or exciton effects? The Journal of Chemical Physics 2010, 133, 094701.

(35)Misawa, K.; Kobayashi, T., Ultrafast exciton and excited-exciton dynamics in J-aggregates of three-level porphyrin molecules. The Journal of Chemical Physics 1999, $110,5844-5850$.

(36)Kano, H.; Saito, T.; Kobayashi, T., Dynamic intensity borrowing in porphyrin J-aggregates revealed by sub-5-fs spectroscopy. The journal of physical chemistry B 2001, 105, 413-419.

(37)Kano, H.; Kobayashi, T., Time-resolved fluorescence and absorption spectroscopies of porphyrin J-aggregates. The Journal of Chemical Physics 2002, 116, 184.

(38) Kelbauskas, L.; Bagdonas, S.; Dietel, W.; Rotomskis, R., Excitation relaxation and structure of TPPS4 J-aggregates. Journal of Luminescence 2003, 101, 253-262.

(39) Miura, A.; Shibata, Y.; Chosrowjan, H.; Mataga, N.; TAMAI, N., Feratosecond fluorescence spectroscopy and near-field spectroscopy of water-soluble tetra(4-sulfonatophenyl)porphyrin and its J-aggregate. Journal Of Photochemistry And Photobiology A-Chemistry 2006, 178, 192-200.

(40)Gulbinas, V.; Karpicz, R.; Augulis, R.; Rotomskis, R., Exciton relaxation in nanotubular TPPS4 aggregates in water solution and in polymeric matrix. Chemical Physics 2007, 332, 255-261.

(41)Kuciauskas, D.; Kiskis, J.; Caputo, G. A.; Gulbinas, V., Exciton Annihilation and Energy Transfer in Self-Assembled Peptide-Porphyrin Complexes Depends on Peptide Secondary Structure. The journal of physical chemistry B 2010, 114, 16029-16035.

(42)Snellenburg, J. J.; Laptenok, S. P.; Seger, R.; Mullen, K. M.; van Stokkum, I. H., Glotaran: a Java-based graphical user interface for the R package TIMP. J. Stat. Softw 2012, 49, 1-22.

(43)Philpott, M. R., Theory of the Coupling of Electronic and Vibrational Excitations in Molecular Crystals and Helical Polymers. The Journal of Chemical Physics 1971, 55, 
2039.

(44)Lemaistre, J. P., Energy relaxation of exciton states in molecular aggregates. Journal of Luminescence 1998, 76-77, 437-440.

(45)Bednarz, M.; Malyshev, V. A.; Knoester, J., Intraband relaxation and temperature dependence of the fluorescence decay time of one-dimensional Frenkel excitons: The Pauli master equation approach. The Journal of Chemical Physics 2002, 117, 6200-6213. (46)Heijs, D.; Malyshev, V.; Knoester, J., Decoherence of Excitons in Multichromophore Systems: Thermal Line Broadening and Destruction of Superradiant Emission. Physical Review Letters 2005, 95, 177402.

(47) Augulis, R.; Malyshev, A. V.; Malyshev, V. A.; Pugzlys, A.; Knoester, J.; van Loosdrecht, P. H. M., Quest for Order in Chaos: Hidden Repulsive Level Statistics in Disordered Quantum Nanoaggregates. Journal Of Physical Chemistry Letters 2010, 1, 2911-2916.

(48)Leegwater, J.; Mukamel, S., Exciton-scattering mechanism for enhanced nonlinear response of molecular nanostructures. Physical Review A 1992, 46, 452-464.

(49)Juzeliūnas, G.; Knoester, J., Pump-probe spectrum of molecular assemblies of arbitrary structure and dimension. The Journal of Chemical Physics 2000, 112, 2325.

(50)Bednarz, M.; Knoester, J., The linear absorption and pump-probe spectra of cylindrical molecular aggregates. The journal of physical chemistry B 2001, 105, 12913-12923.

(51)Didraga, C.; Klugkist, J. A.; Knoester, J., Optical Properties of Helical Cylindrical Molecular Aggregates: The Homogeneous Limit. The journal of physical chemistry $B$ 2002, 106, 11474-11486.

(52)Kaiser, T. E.; Scheblykin, I. G.; Thomsson, D.; Würthner, F., Temperature-dependent exciton dynamics in J-aggregates-when disorder plays a role. The journal of physical chemistry B 2009, 113, 15836-15842.

(53)Fidder, H.; Knoester, J.; Wiersma, D. A., Optical properties of disordered molecular aggregates: A numerical study. The Journal of Chemical Physics 1991, 95, 7880-7890.

(54) Malyshev, V.; Moreno, P., Hidden structure of the low-energy spectrum of a one-dimensional localized Frenkel exciton. Physical Review B 1995, 51, 14587-14593.

(55)Gülen, D., Significance of the Excitonic Intensity Borrowing in the J-/H-aggregates of Bacteriochlorophylls/Chlorophylls. Photosynthesis Research 2006, 87, 205-214.

(56) To effectively account for the effects of vibrations on the peak positions, the monomer excitation energies were taken as $1.84 \mathrm{eV}$ and $2.87 \mathrm{eV}$ for the $\mathrm{Q}$ and $\mathrm{B}$ bands, respectively, compared to $1.87 \mathrm{eV}$ and $2.85 \mathrm{eV}$ for the linear spectra. To account for line broadening, a homogeneous linewidth of $0.03 \mathrm{eV}$ was phenomenologically added to all transitions.

(57)Fidder, H.; Knoester, J.; Wiersma, D., Observation of the one-exciton to two-exciton transition in a J aggregate. The Journal of Chemical Physics 1993, 98, 6564.

(58)Pugzlys, A.; Augulis, R.; van Loosdrecht, P. H. M.; Didraga, C.; Malyshev, V. A.; Knoester, J., Temperature-dependent relaxation of excitons in tubular molecular 
aggregates: Fluorescence decay and stokes shift. The journal of physical chemistry $B$ 2006, 110, 20268-20276.

(59)Bednarz, M.; Malyshev, V.; Knoester, J., Temperature Dependent Fluorescence in Disordered Frenkel Chains: Interplay of Equilibration and Local Band-Edge Level Structure. Physical Review Letters 2003, 91, 217401.

(60) Scheblykin, I. G.; Sliusarenko, O. Y.; Lepnev, L. S.; Vitukhnovsky, A. G.; Van der Auweraer, M., Excitons in Molecular Aggregates of 3,3'-Bis-[3-sulfopropyl]-5,5'-dichloro-9- ethylthiacarbocyanine (THIATS): Temperature Dependent Properties. The journal of physical chemistry B 2001, 105, 4636-4646.

(61) van Stokkum, I. H. M.; Larsen, D. S.; Van Grondelle, R., Global and target analysis of time-resolved spectra. Biochimica Et Biophysica Acta 2004, 1657, 82-104. 